Supporting Information

\title{
Significance of Dopant/Component Miscibility to Efficient N-Doping in Polymer Solar Cells
}

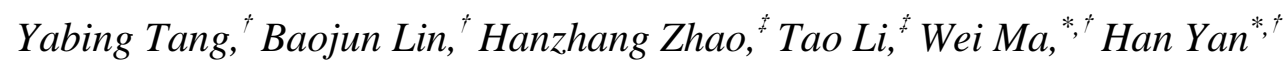

†State Key Laboratory for Mechanical Behavior of Materials, Xi’an Jiaotong

University, Xi'an 710049, P. R. China

${ }^{\ddagger}$ Center of Spintronics and Quantum Systems, State Key Laboratory for Mechanical

Behavior of Materials, School of Materials Science and Engineering, Xi'an Jiaotong

University, Xi'an 710049, P. R. China

*Email: mseyanhan@xjtu.edu.cn

*Email: $\underline{\text { msewma@xjtu.edu.cn }}$ 


\section{Computational details about the ternary phase diagrams.}

According to the lattice-based hypothesis for molecular chain fluids, a simple model to describe the film formation in the ternary system was given by Altena. ${ }^{1}$ The free energy of mixing is given by the equation:

$$
\frac{\Delta G_{M}}{R T}=n_{1} \ln \varphi_{1}+n_{2} \ln \varphi_{2}+n_{3} \ln \varphi_{3}+\chi_{12} n_{1} \varphi_{2}+\chi_{13} n_{1} \varphi_{3}+\chi_{23} N_{2} n_{2} \varphi_{3}
$$

where the subscript $\mathrm{M}$ is a symbol of mixing process, $\mathrm{R}$ is the universal gas constant, $n_{i}$ is the number of moles and $\varphi_{i}$ is volume fraction of component $\mathrm{i} \in$ $\{1,2,3\} \mid\{1=\mathrm{CB}, 2=$ Polymer, $3=\mathrm{N}-\mathrm{DMBI}\}, N_{i}$ (Table S2) is the number of lattice occupied by one molecular of component $\mathrm{i}$ which is calculated by the molecular weight determined by gel permeation chromatography (GPC) (Figure S7) (PCE10 : $120 \mathrm{kDa}, \mathrm{PBDB}-\mathrm{T}: 68 \mathrm{kDa}, \mathrm{N} 2200: 185 \mathrm{kDa}$ ) and segment molar ratio (hence, $N_{1}=1$, $N_{2}=\frac{\text { molecular weight of polymer }}{\text { molecular weight of segment }} \times \frac{\text { molar volume of segment }}{\text { molar volume of } C B} \quad, \quad N_{3}=$ $\left.\frac{\text { molar volume of } N-D M B I}{\text { molar volume of } C B}\right) . \chi_{i j}$ is the Flory-Huggins interaction parameter between component i and j (Table S2). The Flory-Huggins interaction parameters are calculated by Hildebrand solubility parameters for materials with similar polarity according to the expression: $:^{2-4}$

$$
\chi_{i j-\text { Hildebrand }}=\frac{v_{1}}{R T}\left(\delta_{i}-\delta_{j}\right)^{2}+0.34
$$

or by Hansen solubility parameters according to the expression: ${ }^{5-7}$

$$
\chi_{i j-\text { Hansen }}=\frac{\alpha v_{1}}{R T}\left[\left(\delta_{i, D}-\delta_{j, D}\right)^{2}+\frac{1}{4}\left(\delta_{i, P}-\delta_{j, P}\right)^{2}+\frac{1}{4}\left(\delta_{i, H}-\delta_{j, H}\right)^{2}\right]
$$


Where $v_{1}$ is the molar volume of $\mathrm{CB}, 0.34$ is an empirical value of entropic contribution, ${ }^{2-4}$ and the precise value adopted is not critical. $\alpha$ is an adjustable parameter related to the polymer length (that varies from 0.5 to 1 ), and Lindvig et al. have empirically determined $\alpha$ to be 0.6 for a portion of polymers. ${ }^{7} \delta_{i, D} \delta_{i, P}$ and $\delta_{i, H}$ represent the parts of Hansen's total solubility parameters for dispersion forces, permanent dipole-permanent dipole forces and hydrogen bonding, which are calculated by the HSPiP software via Yamamoto-Molecular Break (Y-MB) method, ${ }^{8-11} \delta_{i}$ is the total solubility parameter of component $\mathrm{i}$, which is given by: $:^{5-7,12}$

$$
\delta_{i-\text { Hansen }}=\sqrt{\delta_{i, D}^{2}+\delta_{i, P}^{2}+\delta_{i, H}^{2}}
$$

or by: ${ }^{2-4}$

$$
\delta_{i-\text { Hildebrand }}=K \sqrt{\gamma_{i}}
$$

$\gamma_{i}$ is the surface energy calculated by contact angle (Figure 1d-f and Figure S2) via Neumann's method, ${ }^{13}$ and $\mathrm{K}$ is a proportionality constant simply given by $116 \times$ $10^{3} m^{-\frac{1}{2}}{ }^{2-4}$ The Y-MB method provides an automatic way of calculating the $\delta_{D}, \delta_{P}, \delta_{H}$ and other parameters (e.g. density, molar mass, molar volume, etc.) through simplified 3D molecule input. ${ }^{8-10}$ To confirm the accuracy of the calculated values, the experiment determined $\delta_{i \text {-Hildebrand }}$ (Table S1) by surface energy is given to compare with $\delta_{i-\text { Hansen }}$ (Table S1) because the Hansen's total solubility parameter should be equal to the Hildebrand solubility parameter. ${ }^{12,14}$ The same orders of $\delta_{i-H i l d e b r a n d}$ and $\delta_{i-\text { Hansen }}$ enable us to use the calculated Hansen solubility parameters to perform follow-up analysis. The relative orders of $\chi_{i j-H i l d e b r a n d}$ and $\chi_{i j-H a n s e n}$ are similar 
(Table S2). Nevertheless, the ternary phase diagrams calculated by $\chi_{i j-\text { Hansen }}$ (Figure S6) are far from the experimental observations (Figure 3g-i). Phase separation occurs between N-DMBI and PBDB-T at 10 wt $\%$ N-DMBI content, while the composition point of $10 \mathrm{wt} \% \mathrm{~N}-\mathrm{DMBI}$ doped PBDB-T films is located in the single phase region (Figure S6b). Thus we draw the ternary phase diagrams calculated by $\chi_{i j-H i l d e b r a n d}$ (Figure 3a-c).

The binodal line is the boundary between the single phase region and the metastable region. It defines the onset of phase separation. Using the definition of the chemical potentials: $:^{2,4}$

$$
\Delta \mu_{i}=\frac{\partial \Delta G_{M}}{\partial n_{i}}
$$

The binodal line is calculated according to the following equations:

$$
\begin{aligned}
& \frac{\Delta \mu_{1}}{R T}=\ln \varphi_{1}+\left(1-\varphi_{1}\right)-\frac{\varphi_{2}}{N_{2}}-\frac{\varphi_{3}}{N_{3}}+\left(\chi_{12} \varphi_{2}+\chi_{13} \varphi_{3}\right)\left(\varphi_{2}+\varphi_{3}\right)-\chi_{23} \varphi_{2} \varphi_{3} \\
& \frac{\Delta \mu_{2}}{R T}=\ln \varphi_{2}+\left(1-\varphi_{2}\right)-\varphi_{1} N_{2}-\frac{\varphi_{3} N_{2}}{N_{3}}+\left(X_{12} \varphi_{1} N_{2}+\chi_{23} N_{2} \varphi_{3}\right)\left(\varphi_{1}+\varphi_{3}\right) \\
&-X_{13} \varphi_{1} \varphi_{3} N_{2} \\
& \frac{\Delta \mu_{3}}{R T}=\ln \varphi_{3}+\left(1-\varphi_{3}\right)-\varphi_{1} N_{3}-\frac{\varphi_{2} N_{3}}{N_{2}}+\left(\chi_{13} \varphi_{1} N_{3}+\chi_{23} \varphi_{2} N_{3}\right)\left(\varphi_{1}+\varphi_{2}\right) \\
&-\chi_{12} \varphi_{1} \varphi_{2} N_{3} \\
& \Delta \mu_{i, A}=\Delta \mu_{i, B} \\
& \varphi_{1, A}+\varphi_{2, A}+\varphi_{3, A}=\varphi_{1, B}+\varphi_{2, B}+\varphi_{3, B}=1
\end{aligned}
$$


The tie lines connect the compositions with equal chemical potentials. The region inside the spinodal line is completely unstable, it can be evaluated from the relation:

$$
\frac{\partial^{2}\left(\Delta G_{M}\right)}{\partial \varphi_{2}^{2}} \frac{\partial^{2}\left(\Delta G_{M}\right)}{\partial \varphi_{3}^{2}}-\frac{\partial^{2}\left(\Delta G_{M}\right)}{\partial \varphi_{2} \partial \varphi_{3}} \frac{\partial^{2}\left(\Delta G_{M}\right)}{\partial \varphi_{2} \partial \varphi_{3}}=0
$$

The Gibbs free energy can be transformed to the following equation: ${ }^{15}$

$$
\frac{\Delta G_{M}}{R T n_{t o t}}=\varphi_{1} \ln \varphi_{1}+\frac{\varphi_{2} \ln \varphi_{2}}{N_{2}}+\frac{\varphi_{3} \ln \varphi_{3}}{N_{3}}+\chi_{12} \varphi_{1} \varphi_{2}+\chi_{13} \varphi_{1} \varphi_{3}+\chi_{23} \varphi_{2} \varphi_{3}
$$

The second order derivatives of the Gibbs free energy are as follows:

$$
\begin{gathered}
\frac{\partial^{2}\left(\frac{\Delta G_{M}}{R T n_{t o t}}\right)}{\partial \varphi_{2}^{2}}=\frac{1}{\varphi_{1}}+\frac{1}{\varphi_{2} N_{2}}-2 \chi_{12} \\
\frac{\partial^{2}\left(\frac{\Delta G_{M}}{R T n_{t o t}}\right)}{\partial \varphi_{3}^{2}}=\frac{1}{\varphi_{1}}+\frac{1}{\varphi_{3} N_{3}}-2 \chi_{13} \\
\frac{\partial^{2}\left(\frac{\Delta G_{M}}{R T n_{t o t}}\right)}{\partial \varphi_{2} \partial \varphi_{3}}=\frac{1}{\varphi_{1}}+\chi_{23}-\chi_{13}-\chi_{12}
\end{gathered}
$$


Table S1. Parameters for calculation of the Flory-Huggins interaction parameters $(\chi)$ and ternary phase diagram. For the polymer, molar volume $\left(\mathrm{V}_{\mathrm{m}}\right)$ and the molecular weight $\left(\mathrm{M}_{\mathrm{wt}}\right)$ belong to the segment.

\begin{tabular}{ccccccccc}
\hline Materials & $\begin{array}{c}\mathrm{V}_{\mathrm{m}} \\
\left(\mathrm{cm}^{3} / \mathrm{mol}\right)\end{array}$ & $\begin{array}{c}\mathrm{M}_{\mathrm{wt}} \\
(\mathrm{g} / \mathrm{mol})\end{array}$ & $\begin{array}{c}\delta_{i, D} \\
\left(\mathrm{MPa}^{1 / 2}\right)\end{array}$ & $\begin{array}{c}\delta_{i, P} \\
\left(\mathrm{MPa}^{1 / 2}\right)\end{array}$ & $\begin{array}{c}\delta_{i, H} \\
\left(\mathrm{MPa}^{1 / 2}\right)\end{array}$ & $\begin{array}{c}\delta_{i-\text { Hansen }} \\
\left(\mathrm{MPa}^{1 / 2}\right)\end{array}$ & $\begin{array}{c}Y_{i} \\
(\mathrm{mN} / \mathrm{m})\end{array}$ & $\begin{array}{c}\delta_{i-\text { Hildebrand }} \\
\left(\mathrm{MPa}^{1 / 2}\right)\end{array}$ \\
\hline PCE10 & 746 & 891.4 & 19.5 & 3.5 & 6.8 & 20.9 & $34.6 \pm 0.6 ; 32.0^{16}$ & 21.6 \\
\hline PBDB-T & 985.9 & 1185.9 & 20.7 & 5.1 & 7.3 & 22.6 & $35.3 \pm 0.3 ; 35.7^{17}$ & 21.8 \\
\hline N2200 & 954.1 & 991.5 & 19.8 & 1.4 & 3.1 & 20.1 & $32.4 \pm 0.3$ & 20.9 \\
\hline CB & 102.1 & 112.0 & 19.0 & 4.3 & 2.0 & $19.6^{14}$ & & \\
\hline N-DMBI & 236.1 & 267.4 & 20.4 & 9.8 & 6.8 & 23.6 & & \\
\hline
\end{tabular}

Table S2. Flory-Huggins interaction parameters ( $\chi_{i j-H i l d e b r a n d}$ and $\left.\chi_{i j-H a n s e n}\right)$ obtained by calculated Hansen solubility parameters via Y-MB method, and the degree of polymerization $\left(\mathrm{N}_{\mathrm{i}}\right)$ for the polymers and N-DMBI. Here, $\alpha$ is simply adopted by 0.6.

\begin{tabular}{|c|c|c|c|c|c|c|c|c|c|c|}
\hline \multicolumn{3}{|c|}{ Material Systems } & $X_{12}$ & $X_{13}$ & $X_{23}$ & $X_{12}$ & $X_{13}$ & $X_{23}$ & \multirow{2}{*}{$\mathrm{N}_{2}$} & \multirow{2}{*}{$\mathrm{N}_{3}$} \\
\hline 1 & 2 & 3 & \multicolumn{3}{|c|}{$\chi_{i j-\text { Hildebrand }}$} & \multicolumn{3}{|c|}{$\chi_{i j-H a n s e n}$} & & \\
\hline CB & PCE10 & N-DMBI & 0.41 & 1.00 & 0.64 & 0.15 & 0.38 & 0.27 & 983 & 2 \\
\hline $\mathrm{CB}$ & PBDB-T & N-DMBI & 0.71 & 1.00 & 0.38 & 0.25 & 0.38 & 0.14 & 554 & 2 \\
\hline CB & N2200 & N-DMBI & 0.35 & 1.00 & 0.84 & 0.08 & 0.38 & 0.53 & 1744 & 2 \\
\hline
\end{tabular}

Table S3. Photovoltaic performances of PCE10/N2200 PHJ devices with film thickness of $20 \mathrm{~nm}$ for each layer. Each statistical data is based on 10 independent devices.

\begin{tabular}{|c|c|c|c|c|c|c|c|}
\hline \multicolumn{2}{|c|}{$\begin{array}{c}\text { Materials } \\
\text { (PCE10/N2200) }\end{array}$} & $\begin{array}{c}V_{\text {oc } \max } \\
\text { (V) }\end{array}$ & $\begin{array}{c}\mathrm{FF}_{\max } \\
(\%)\end{array}$ & $\begin{array}{c}J_{s c \max } \\
\left(\mathrm{mA} / \mathrm{cm}^{2}\right)\end{array}$ & $\begin{array}{c}J_{s c \text { avg }} \\
\left(\mathrm{mA} / \mathrm{cm}^{2}\right)\end{array}$ & $\begin{array}{c}\mathrm{PCE}_{\max } \\
(\%)\end{array}$ & $\begin{array}{c}\mathrm{PCE}_{\text {avg }} \\
(\%)\end{array}$ \\
\hline \multirow{6}{*}{$\begin{array}{l}\text { N-DMBI } \\
\text { in } \\
\text { N2200 }\end{array}$} & Control & 0.81 & 66.7 & 1.30 & $1.31 \pm 0.02$ & 0.70 & $0.69 \pm 0.01$ \\
\hline & $0.01 w t \%$ & 0.80 & 62.5 & 1.38 & $1.29 \pm 0.06$ & 0.69 & $0.67 \pm 0.01$ \\
\hline & $0.05 w t \%$ & 0.81 & 64.6 & 1.44 & $1.40 \pm 0.05$ & 0.76 & $0.72 \pm 0.02$ \\
\hline & $0.3 w t \%$ & 0.82 & 58.3 & 1.54 & $1.47 \pm 0.06$ & 0.74 & $0.71 \pm 0.02$ \\
\hline & $0.5 w t \%$ & 0.82 & 56.2 & 1.56 & $1.46 \pm 0.05$ & 0.72 & $0.69 \pm 0.01$ \\
\hline & $1 w t \%$ & 0.82 & 61.8 & 1.38 & $1.36 \pm 0.03$ & 0.71 & $0.69 \pm 0.01$ \\
\hline
\end{tabular}


Table S4. Photovoltaic performances of PBDB-T/N2200 PHJ devices with film thickness of $20 \mathrm{~nm}$ for each layer. Each statistical data is based on 10 independent devices.

\begin{tabular}{cccccccc}
\hline $\begin{array}{c}\text { Materials } \\
(\mathrm{PBDB}-\mathrm{T} / \mathrm{N} 2200)\end{array}$ & $\begin{array}{c}V_{\text {oc } \max } \\
(\mathrm{V})\end{array}$ & $\begin{array}{c}\mathrm{FF}_{\max } \\
(\%)\end{array}$ & $\begin{array}{c}\mathrm{J}_{\text {sc } \max } \\
\left(\mathrm{mA} / \mathrm{cm}^{2}\right)\end{array}$ & $\begin{array}{c}\mathrm{J}_{\text {sc avg }} \\
\left(\mathrm{mA} / \mathrm{cm}^{2}\right)\end{array}$ & $\begin{array}{c}\mathrm{PCE}_{\max } \\
(\%)\end{array}$ & $\begin{array}{c}\mathrm{PCE}_{\text {avg }} \\
(\%)\end{array}$ \\
\hline \multirow{3}{*}{$\begin{array}{c}\text { N-DMBI } \\
\text { in }\end{array}$} & 0.78 & 67.3 & 0.40 & $0.41 \pm 0.01$ & 0.21 & $0.21 \pm 0.01$ \\
\cline { 2 - 8 } $\mathrm{N} 2200$ & $0.01 \mathrm{wt} \%$ & 0.80 & 63.9 & 0.43 & $0.40 \pm 0.02$ & 0.22 & $0.20 \pm 0.01$ \\
\cline { 2 - 8 } & $0.05 \mathrm{wt} \%$ & 0.80 & 59.7 & 0.51 & $0.45 \pm 0.03$ & 0.25 & $0.22 \pm 0.01$ \\
\cline { 2 - 8 } & $0.3 \mathrm{wt} \%$ & 0.81 & 57.3 & 0.48 & $0.43 \pm 0.03$ & 0.22 & $0.20 \pm 0.01$ \\
\cline { 2 - 8 } & $1 \mathrm{wt} \%$ & 0.80 & 59.1 & 0.48 & $0.43 \pm 0.03$ & 0.23 & $0.20 \pm 0.01$ \\
\hline
\end{tabular}

Table S5. Photovoltaic performances of PCE10/N2200 PHJ devices with film thickness of $20 \mathrm{~nm}$ for each layer. Each statistical data is based on 6 independent devices.

\begin{tabular}{|c|c|c|c|c|c|c|c|}
\hline \multicolumn{2}{|c|}{$\begin{array}{c}\text { Materials } \\
\text { (PCE10/N2200) }\end{array}$} & $\begin{array}{l}V_{\text {oc max }} \\
(V)\end{array}$ & $\begin{array}{c}\mathrm{FF}_{\text {max }} \\
(\%)\end{array}$ & $\begin{array}{c}J_{s c \max } \\
\left(\mathrm{mA} / \mathrm{cm}^{2}\right)\end{array}$ & $\begin{array}{c}J_{\text {sc avg }} \\
\left(\mathrm{mA} / \mathrm{cm}^{2}\right)\end{array}$ & $\begin{array}{c}\mathrm{PCE}_{\max } \\
(\%)\end{array}$ & $\begin{array}{c}\mathrm{PCE}_{\text {avg }} \\
(\%)\end{array}$ \\
\hline \multirow{5}{*}{$\begin{array}{l}\text { N-DMBI } \\
\text { in } \\
\text { PCE10 }\end{array}$} & Control & 0.79 & 63.0 & 1.17 & $1.14 \pm 0.04$ & 0.58 & $0.55 \pm 0.02$ \\
\hline & $0.01 w t \%$ & 0.78 & 63.2 & 1.14 & $1.15 \pm 0.03$ & 0.57 & $0.53 \pm 0.02$ \\
\hline & $0.05 w t \%$ & 0.79 & 61.0 & 1.18 & $1.13 \pm 0.03$ & 0.57 & $0.54 \pm 0.02$ \\
\hline & $0.3 w t \%$ & 0.78 & 62.5 & 1.13 & $1.12 \pm 0.03$ & 0.56 & $0.54 \pm 0.02$ \\
\hline & $0.5 w t \%$ & 0.79 & 62.2 & 1.15 & $1.09 \pm 0.04$ & 0.56 & $0.54 \pm 0.02$ \\
\hline
\end{tabular}

Table S6. Photovoltaic performances of PCE10/N2200 PHJ devices with film thickness of $50 \mathrm{~nm}$ for each layer. Each statistical data is based on 10 independent devices.

\begin{tabular}{|c|c|c|c|c|c|c|c|}
\hline \multicolumn{2}{|c|}{$\begin{array}{c}\text { Materials } \\
\text { (PCE10/N2200) }\end{array}$} & $\begin{array}{c}V_{\text {oc max }} \\
\text { (V) }\end{array}$ & $\begin{array}{c}\mathrm{FF}_{\text {max }} \\
(\%)\end{array}$ & $\begin{array}{c}J_{s c \max } \\
\left(\mathrm{mA} / \mathrm{cm}^{2}\right)\end{array}$ & $\begin{array}{c}J_{s c \text { avg }} \\
\left(\mathrm{mA} / \mathrm{cm}^{2}\right)\end{array}$ & $\begin{array}{c}\mathrm{PCE}_{\max } \\
(\%)\end{array}$ & $\begin{array}{c}\mathrm{PCE}_{\mathrm{avg}} \\
(\%)\end{array}$ \\
\hline \multirow{5}{*}{$\begin{array}{l}\text { N-DMBI } \\
\text { in } \\
\text { N2200 }\end{array}$} & Control & 0.79 & 62.4 & 0.95 & $0.88 \pm 0.05$ & 0.47 & $0.43 \pm 0.02$ \\
\hline & $0.01 w t \%$ & 0.80 & 63.5 & 0.94 & $0.91 \pm 0.03$ & 0.48 & $0.46 \pm 0.01$ \\
\hline & $0.05 w t \%$ & 0.80 & 58.3 & 1.10 & $1.04 \pm 0.04$ & 0.51 & $0.50 \pm 0.01$ \\
\hline & $0.1 w t \%$ & 0.81 & 61.4 & 1.03 & $0.98 \pm 0.03$ & 0.51 & $0.48 \pm 0.01$ \\
\hline & $0.3 w t \%$ & 0.81 & 61.3 & 0.94 & $0.87 \pm 0.04$ & 0.47 & $0.43 \pm 0.02$ \\
\hline
\end{tabular}


Table S7. Photovoltaic performances of PBDB-T/N2200 PHJ devices with film thickness of $50 \mathrm{~nm}$ for each layer. Each statistical data is based on 10 independent devices.

\begin{tabular}{cccccccc}
\hline $\begin{array}{c}\text { Materials } \\
\text { (PBDB-T/N2200) }\end{array}$ & $\begin{array}{c}V_{\text {oc max }} \\
(\mathrm{V})\end{array}$ & $\begin{array}{c}\mathrm{FF}_{\max } \\
(\%)\end{array}$ & $\begin{array}{c}\mathrm{J}_{\text {sc max }} \\
\left(\mathrm{mA} / \mathrm{cm}^{2}\right)\end{array}$ & $\begin{array}{c}\mathrm{J}_{\text {sc avg }} \\
\left(\mathrm{mA} / \mathrm{cm}^{2}\right)\end{array}$ & $\begin{array}{c}\mathrm{PCE}_{\max } \\
(\%)\end{array}$ & $\begin{array}{c}\mathrm{PCE}_{\text {avg }} \\
(\%)\end{array}$ \\
\hline \multirow{2}{*}{$\begin{array}{c}\text { N-DMBI } \\
\text { in }\end{array}$} & $0.01 \mathrm{wt} \%$ & 0.80 & 55.9 & 0.35 & $0.30 \pm 0.02$ & 0.15 & $0.14 \pm 0.01$ \\
\cline { 2 - 8 } N2200 & $0.05 w t \%$ & 0.79 & 51.9 & 0.38 & $0.35 \pm 0.02$ & 0.16 & $0.14 \pm 0.01$ \\
\cline { 2 - 8 } & $0.1 w t \%$ & 0.79 & 51.9 & 0.34 & $0.32 \pm 0.03$ & 0.14 & $0.13 \pm 0.01$ \\
\cline { 2 - 8 } & $0.3 w t \%$ & 0.78 & 49.5 & 0.33 & $0.31 \pm 0.02$ & 0.13 & $0.12 \pm 0.01$ \\
\hline
\end{tabular}


(a)
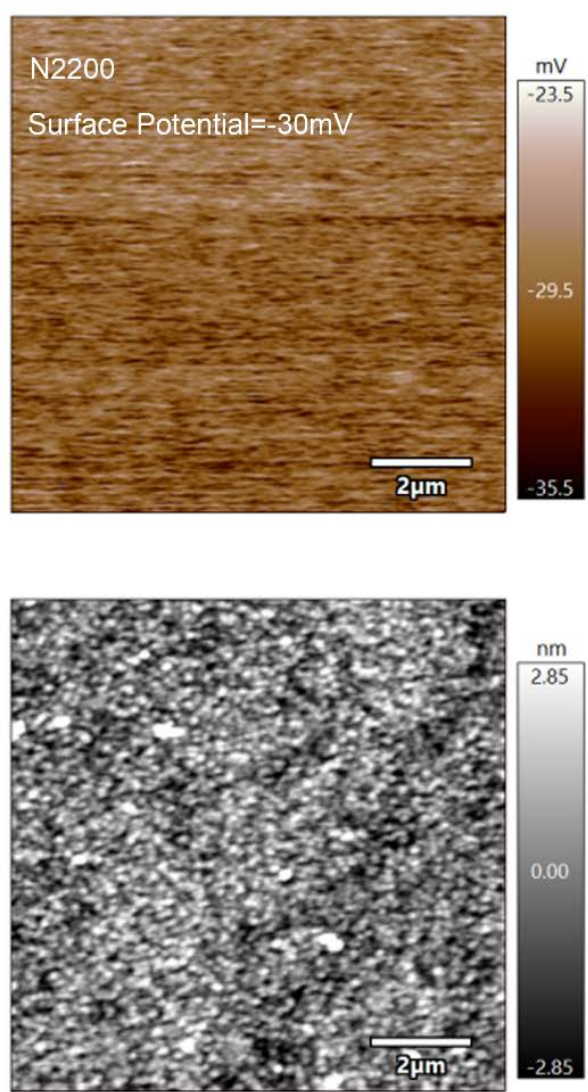

(b)
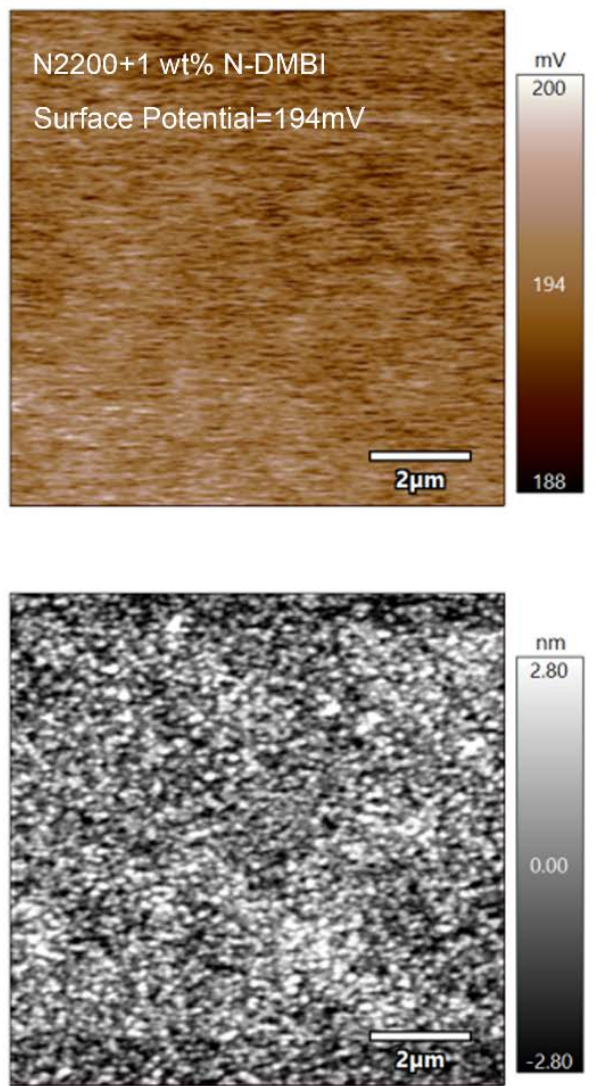

Figure S1. (a) The surface potential and corresponding morphology images of neat N2200 film. (b) The surface potential and corresponding morphology images of 1 wt\% N-DMBI doped N2200 film.

(a)

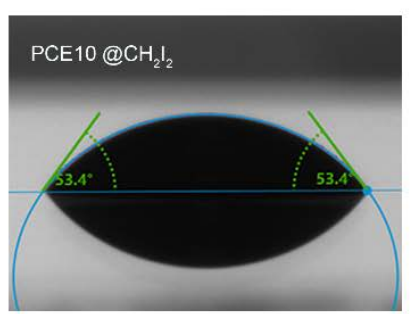

(b)

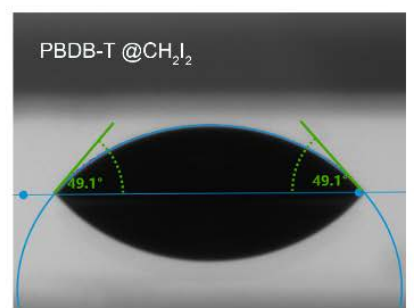

(c)

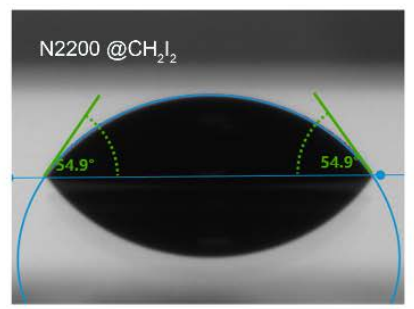

Figure S2. (a-c) Contact angle measurements of PCE10, PBDB-T, and N2200, respectively. 
(a)

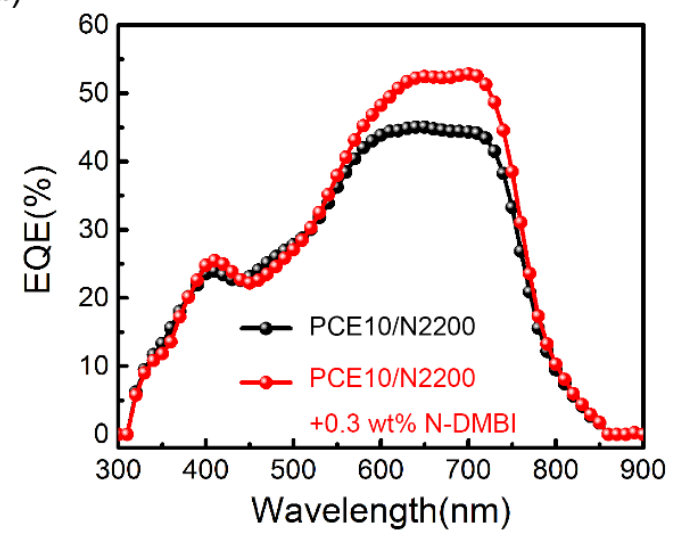

(b)

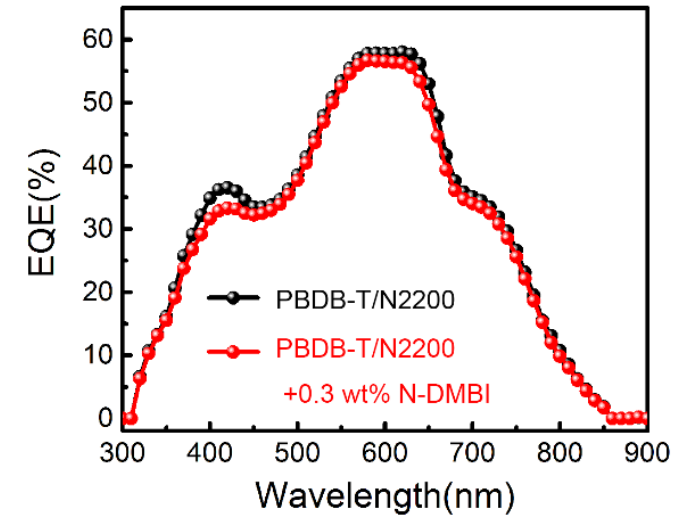

Figure S3. EQE plots of BHJ devices: (a) PCE10/N2200 and (b) PBDB-T/N2200.

(a)
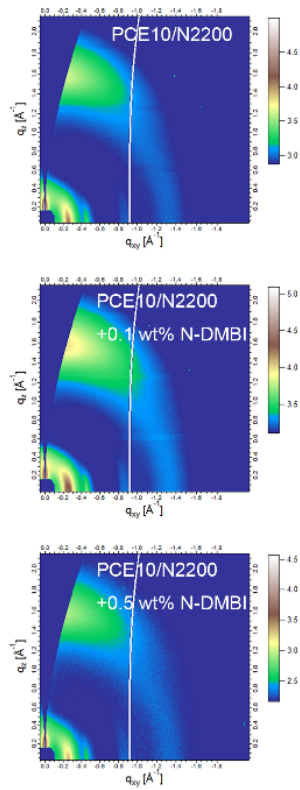

(b)
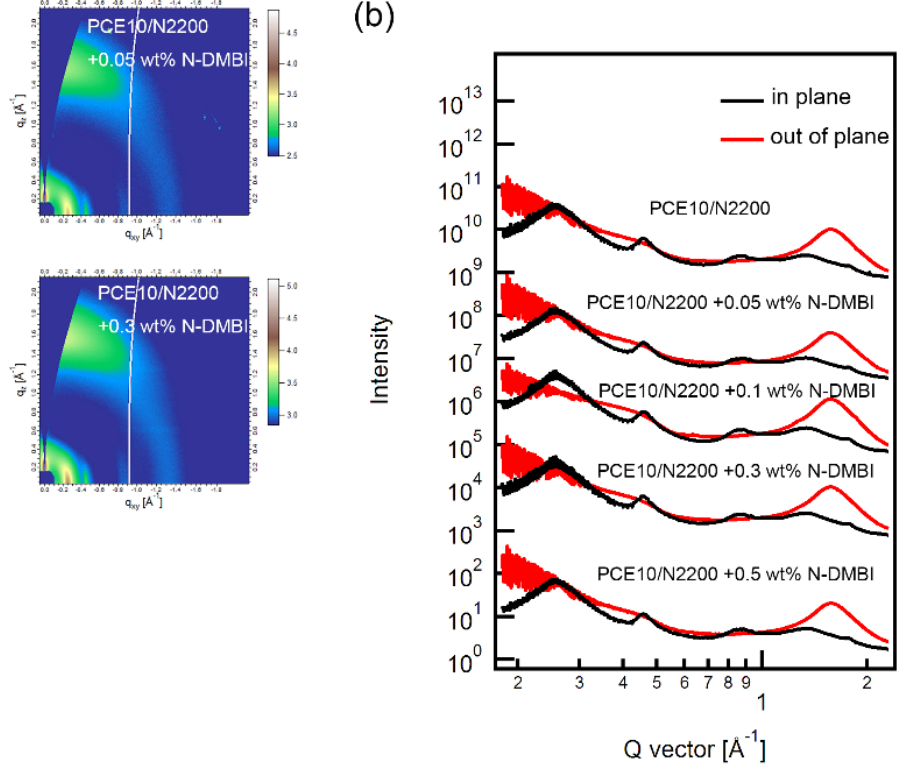

Figure S4. (a) Two-dimensional GIWAXS scattering patterns and (b) the corresponding line profiles of PCE10/N2200 films. 
(a)
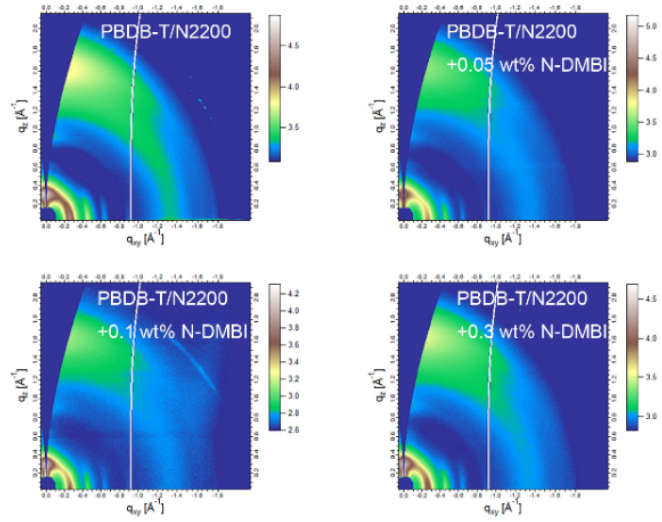

(b)

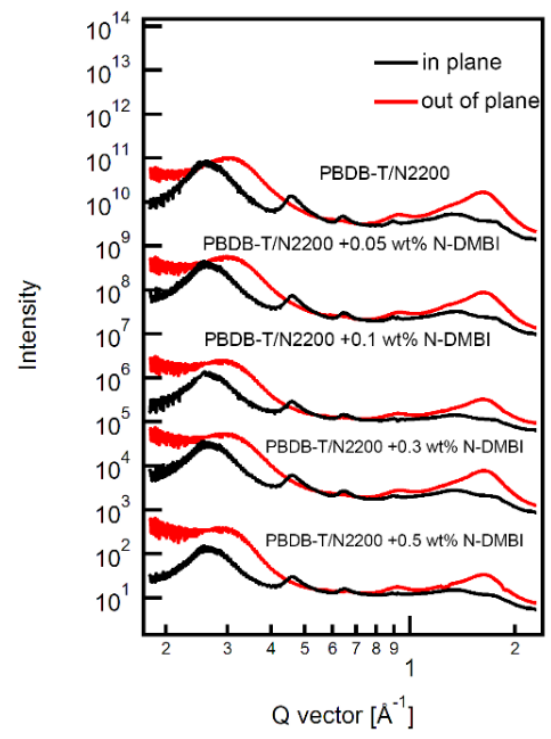

Figure S5. (a) Two-dimensional GIWAXS scattering patterns and (b) the corresponding line profiles of PBDB-T/N2200 films.

(a)

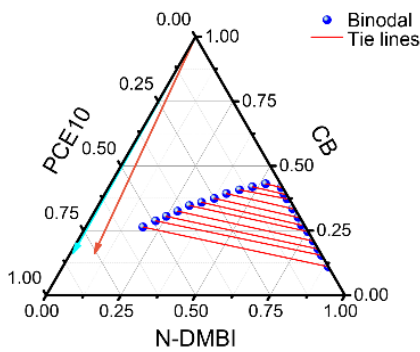

(b)

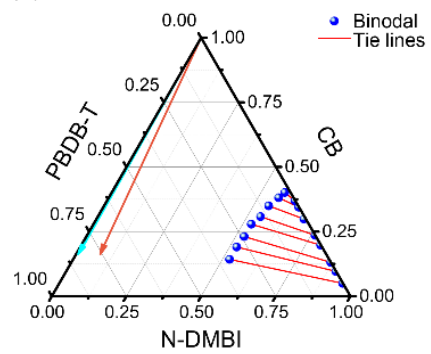

(c)

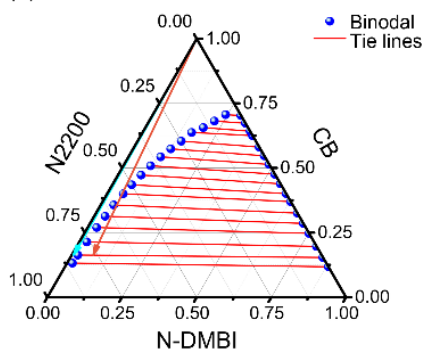

Figure S6. (a-c) Ternary phase diagrams of solvent/polymer/dopant material combinations calculated by $\chi_{i j-\text { Hansen }}$ : (a) CB/PCE10/N-DMBI, (b) CB/PBDB-T/NDMBI, and (c) CB/N2200/N-DMBI. 
(a)

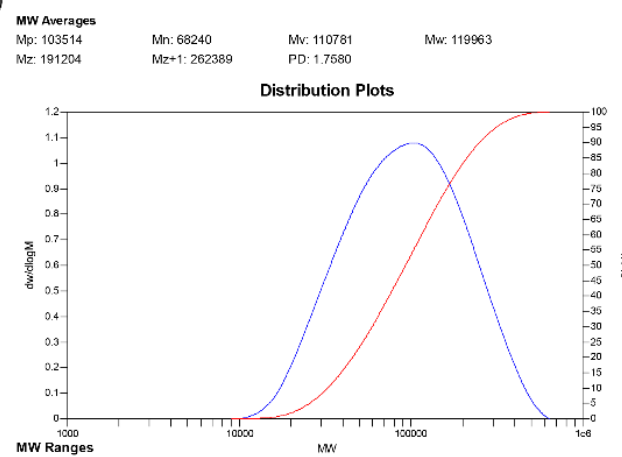

(b)

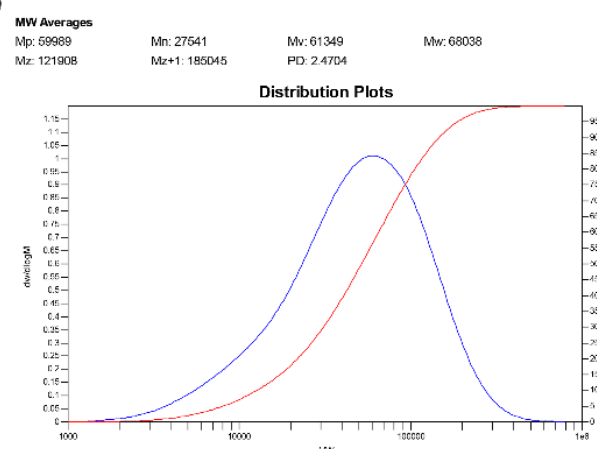

(c)

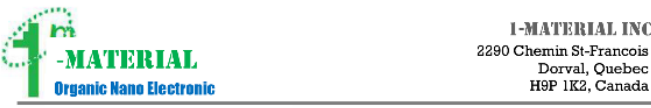

CERTIFICATE OF ANALYSIS

Product:

Application:

CAS $\#$

Chemical Name:

ND120D-12): PNDI-2T; N2200

Laloratory Research Use ONLY

$1100243-40-0$

Poly|11,2,3,6,7,-hexahydro-2,7-bis(2-0ctyldodccyl)-1,3,6.

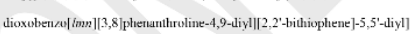

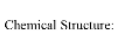

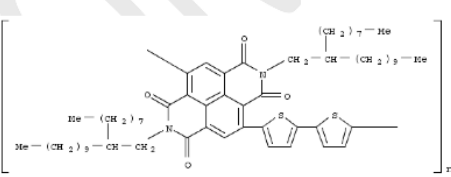

Lot number: $\quad$ YY15042CH

$\begin{array}{ll}\text { Lot number: } & \text { YY15042CH } \\ \text { Appearance: } & \text { Shine deep purple solid }\end{array}$

Volatile moisture balance: $\quad<1.0 \%$

Molecular weight (GPC): $\quad \sim 185,000$ (Mw, PS Standard)

Polydispersity(PDI): 2-3

Solubility:

Soluble in chlorobenzene and dichlorobenzene

NMR analysis:

Structure confirmed

Regularity

Figure S7. (a) Molecular weight of PCE10 determined by GPC. (b) Molecular weight of PBDB-T determined by GPC. (c) Molecular weight of N2200 given by the vendor.

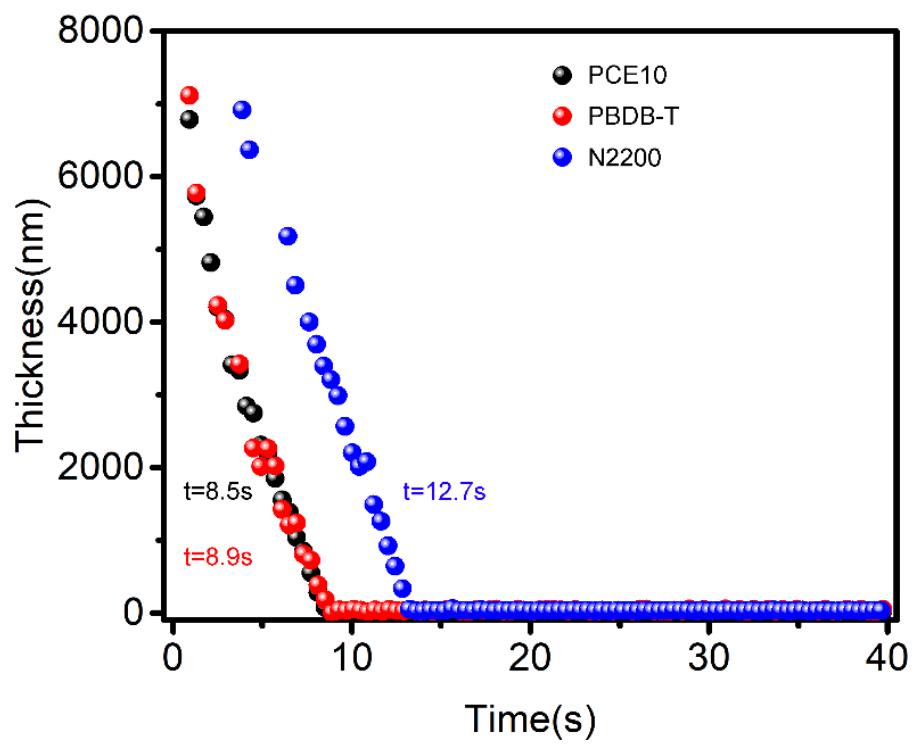

Figure S8. Film formation time of polymer films by in-situ measurement of film thickness. 
(a)

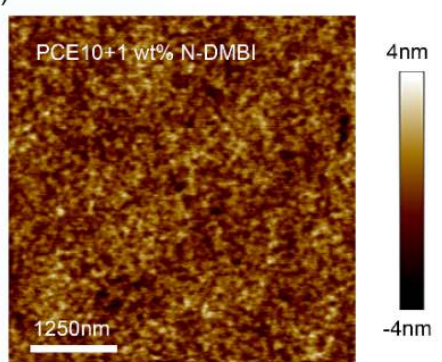

(b)

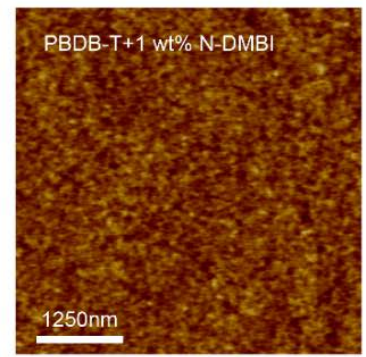

(c)

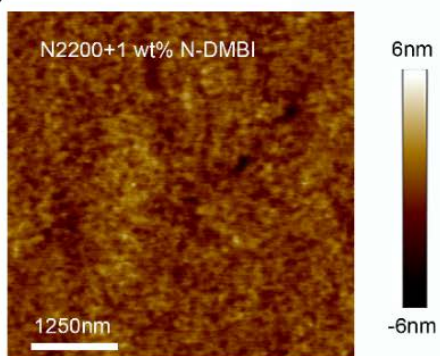

Figure S9. (a-c) Contact-mode atomic force microscopy images of $1 \mathrm{wt} \% \mathrm{~N}-\mathrm{DMBI}$ doped PCE10, PBDB-T and N2200 films, respectively. 


\section{REFERENCES}

(1) Altena, F. W.; Smolders, C. A. Calculation of Liquid Liquid-Phase Seperation in

a Ternary-System of a Polymer in a Mixture of a Solvent and a Nonsolvent. Macromolecules 1982, 15, 1491-1497.

(2) Kim, J.-H.; $\quad$ Gadisa, A.; $\quad$ Schaefer, C.; $\quad$ Yao, H.; $\quad$ Gautam, B. R.; $\quad$ Balar, N.; Ghasemi, M.; Constantinou, I.; So, F.; O'Connor, B. T.; Gundogdu, K.; Hou, J.; Ade, H. Strong Polymer Molecular Weight-Dependent Material Interactions: Impact on the Formation of the Polymer/Fullerene Bulk Heterojunction Morphology. Journal of Materials Chemistry A 2017, 5, 13176-13188.

(3) Kouijzer, S.; Michels, J. J.; van den Berg, M.; Gevaerts, V. S.; Turbiez, M.;

Wienk, M. M.; Janssen, R. A. J. Predicting Morphologies of Solution Processed Polymer:Fullerene Blends. J. Am. Chem. Soc. 2013, 135, 12057-12067.

(4) Nilsson, S.; Bernasik, A.; Budkowski, A.; Moons, E. Morphology and Phase Segregation of Spin-Casted Films of Polyfluorene/PCBM Blends. Macromolecules 2007, 40, 8291-8301.

(5) Leman, D.; Kelly, M. A.; Ness, S.; Engmann, S.; Herzing, A.; Snyder, C.; Ro, H. W.; Kline, R. J.; DeLongchamp, D. M.; Richter, L. J. In Situ Characterization of Polymer-Fullerene Bilayer Stability. Macromolecules 2015, 48, 383-392. 
(6) Bokel, F. A.; Engmann, S.; Herzing, A. A.; Collins, B. A.; Ro, H. W.;

DeLongchamp, D. M.; Richter, L. J.; Schaible, E.; Hexemer, A. In Situ X-ray Scattering Studies of the Influence of an Additive on the Formation of a Low-Bandgap Bulk Heterojunction. Chemistry of Materials 2017, 29, 2283-2293.

(7) Lindvig, T.; Michelsen, M. L.; Kontogeorgis, G. M.. A Flory-Huggins Model Based on the Hansen Solubility Parameters. Fluid Phase Equilib 2002, 203, 247-260.

(8) Stefanis E.; Panayiotou C. Prediction of Hansen Solubility Parameters with a New Group-Contribution Method. Int J Thermophys 2018, 29, 568-585.

(9) Filly A.; Fabiano-Tixier A.; Lemasson Y.; Roy C.; Fernandez X.; Chemat F. Extraction of Aroma Compounds in Blackcurrent Buds by Alternative Solvents: Throretical and Experimental Solubility Study. Comptes Rendus Chimie 2014, 17, 1268-1275.

(10) Li Y.; Fabiano-Tixier AS.; Chemat F. Essential Oils as Reagents in Green Chemistry. In: Essential Oils as Reagents in Green Chemistry. SpringerBriefs in Molecular Science. Springer, Cham 2014.

(11) Abbott, S. J.; Hansen, C. M. Hansen Solubility Parameters in Practice (software) 5th Edition, www.hansen-solubility.com, 2015.

(12) Zhang, C. Influence of Microstructure on Thermo- and Photo-stability in Organic Bulk-heterojunction Solar Cell. Friedrich-Alexander-Universität ErlangenNürnberg 2018. 
(13) Żenkiewicz, M. Methods for the Calculation of Surface Free Energy of Solids. J. Achiev. Mater. Manuf. Eng. 2007, 24, 137-145.

(14) Hansen, C. M. Hansen Solubility Parameters: A User's Handbook, Second Edition. CRC Press:New York 2007.

(15) Kim, M.; Lee, J.; Jo, SB.; Sin, DH.; Ko, H.; Lee, H.; Lee, SG.; Cho, K. Critical Factors Governing Vertical Phase Separation in Polymer-PCBM Blend Films for Organic Solar Cells. J. Mater. Chem. A 2016, 4, 15522.

(16) Liu, Y.; Tang, D.; Zhang, K.; Huang, P.; Wang, Z.; Zhu, K.; Li, Z.; Yuan, L.; Fan, J.; Zhou, Y.; Song, B. Tuning Surface Energy of Conjugated Polymers via Fluorine Substitution of Side Alkyl Chains: Influence on Phase Separation of Thin Films and Performance of Polymer Solar Cells. ACS Omega 2017, 2, 2489-2498.

(17) Bai, Y.; Yang, B.; Chen, X.; Wang, F.; Hayat, T.; Alsaedi, A.; Tan, Z. Constructing Desired Vertical Component Distribution Within a PBDB-T:ITIC-M Photoactive Layer via Fine-Tuning the Surface Free Energy of a Titanium Chelate Cathode Buffer Layer. Frontiers in Chemistry, 2018, 6, 292. 